DESY 94-088

\title{
Numerical simulations and the strength of the electroweak phase transition
}

\author{
Z. Fodor, J. Hein, K. Jansen, A. Jaster, I. Montvay \\ Deutsches Elektronen-Synchrotron DESY, \\ Notkestr. 85, D-22603 Hamburg, Germany \\ F. Csikor \\ Institute for Theoretical Physics, Eötvös University, \\ Budapest, Hungary
}

May 24, 1994

\begin{abstract}
Numerical simulations are performed to study the finite temperature phase transition in the SU(2) Higgs model on the lattice. The strength of the first order phase transition is investigated by determining the latent heat and the interface tension on $L_{t}=2$ lattices. The values of the Higgs boson mass presently chosen are below 50 $\mathrm{GeV}$. Our results are in qualitative agreement with two-loop resummed perturbation theory.
\end{abstract}

\section{Introduction}

The standard calculational method for the study of the symmetry restoring electroweak phase transition [1] is resummed perturbation theory [2, 3, 4]. The highest known order in the gaugeand scalar quartic coupling is $g^{4}, \lambda^{2}$, combined with a high temperature expansion [5]. In the Higgs phase with broken symmetry perturbation theory is expected to work well for not very high Higgs boson masses, since the couplings are small. The perturbative calculations can be extended to the vicinity of the symmetric phase for $\lambda \ll g^{2}$. In the high temperature symmetric

\footnotetext{
*On leave from Institute for Theoretical Physics, Eötvös University, Budapest, Hungary.
} 
phase, however, the situation is similar to high temperature QCD. Therefore, irreparable infrared singularities occur which prevent a quantitative control of graph resummation [6]. Since the calculation of physical quantities characterizing the phase transition requires the knowledge of both phases, there is a priory no reason why perturbation theory could provide a quantitative treatment of the electroweak phase transition for physically relevant couplings.

An obvious non-perturbative method for the study of the symmetry restoring phase transition is numerical simulation on the lattice. Omitting, for simplicity, fermions and the $U(1)$ gauge field one is left with the $\mathrm{SU}(2)$ Higgs model describing the interaction of a four-component Higgs scalar field with the $\mathrm{SU}(2)$ gauge field. After pioneering works [7, 8], recent numerical simulations contributed a lot to the understanding of the finite temperature behaviour of the $\mathrm{SU}(2)$ Higgs model [9]. Another line of research has also been initiated recently [10, 11]. In this approach one studies the three-dimensional effective theory obtained by dimensional reduction in the high-temperature limit.

An important issue is the order and strength of the phase transition. For instance, the possibility of electroweak baryogenesis [12] requires a strong enough first order electroweak phase transition [13] (for a recent review see [14]). Up to now the four dimensional numerical simulations [9] have been concentrated on relatively heavy Higgs boson masses, where the properties of the phase transition are technically difficult to disentangle. In the numerical approach based on dimensional reduction indications have been found that the phase transition is stronger than given by perturbation theory [15]. In the present letter we investigate this question in the four dimensional SU(2) Higgs model for lighter Higgs masses, below $50 \mathrm{GeV}$. Since this region of parameters of the minimal standard model is already excluded by experiments, our present scope is merely theoretical because we would like to check the validity of some other theoretical approximation schemes, e.g. resummed perturbation theory. We plan to extend this investigation to heavier Higgs boson masses in future papers.

The present letter is our first short communication. Technical details will not be included here, but will be postponed to a subsequent publication with more numerical data [16].

\section{Physical parameters}

The lattice action of the $\mathrm{SU}(2)$ Higgs model is conventionally written as

$$
\begin{gathered}
S[U, \varphi]=\beta \sum_{p l}\left(1-\frac{1}{2} \operatorname{Tr} U_{p l}\right) \\
+\sum_{x}\left\{\frac{1}{2} \operatorname{Tr}\left(\varphi_{x}^{+} \varphi_{x}\right)+\lambda\left[\frac{1}{2} \operatorname{Tr}\left(\varphi_{x}^{+} \varphi_{x}\right)-1\right]^{2}-\kappa \sum_{\mu=1}^{4} \operatorname{Tr}\left(\varphi_{x+\hat{\mu}}^{+} U_{x \mu} \varphi_{x}\right)\right\} .
\end{gathered}
$$

Here $U_{x \mu}$ denotes the $\mathrm{SU}(2)$ gauge link variable and $\varphi_{x}$ is a complex $2 \otimes 2$ matrix in isospin space describing the Higgs scalar field and satisfying

$$
\varphi_{x}^{+}=\tau_{2} \varphi_{x}^{T} \tau_{2}
$$

The bare parameters in the action are $\beta \equiv 4 / g^{2}$ for the gauge coupling, $\lambda$ for the scalar quartic coupling and $\kappa$ for the scalar hopping parameter related to the bare mass square $\mu_{0}^{2}$ by $\mu_{0}^{2}=(1-2 \lambda) / \kappa-8$.

In order to fix the physical parameters in a numerical simulation one has to define and compute some suitable renormalized quantities at zero temperature. The renormalized gauge 
coupling can be determined from the static potential of an external SU(2) charge pair, measured by Wilson loops. The physical Higgs mass $M_{H}$ can be extracted from correlation functions of quantities as the site variable

$$
R_{x} \equiv \frac{1}{2} \operatorname{Tr}\left(\varphi_{x}^{+} \varphi_{x}\right) \equiv \rho_{x}^{2}
$$

or, using $\varphi_{x} \equiv \rho_{x} \alpha_{x}$, the link variables

$$
L_{\varphi} \equiv \frac{1}{2} \operatorname{Tr}\left(\varphi_{x+\hat{\mu}}^{+} U_{x \mu} \varphi_{x}\right), \quad L_{\alpha} \equiv \frac{1}{2} \operatorname{Tr}\left(\alpha_{x+\hat{\mu}}^{+} U_{x \mu} \alpha_{x}\right) .
$$

The W-boson mass $M_{W}$ can be obtained similarly from the composite link fields $(r, k=1,2,3)$

$$
W_{r k} \equiv \frac{1}{2} \operatorname{Tr}\left(\tau_{r} \alpha_{x+\hat{k}}^{+} U_{x k} \alpha_{x}\right)
$$

Since we are interested in the study of the symmetry restoring phase transition on lattices with temporal extension $L_{t}=2$, we have to determine the renormalized parameters at critical points for the $L_{t}=2$ lattices. In order to have a renormalized gauge coupling near the physical value $g_{R}^{2} \simeq 0.5$ we choose $\beta=8$ [17]. As stated before, we would like to have lighter Higgs boson masses than studied in [9]. Therefore we have chosen the two values $\lambda=0.0001$ (referred to as low) and $\lambda=0.0005$ (referred to as high). In these cases the critical hopping parameters turned out to be near $\kappa_{c}^{\text {low }}=0.1283$ and $\kappa_{c}^{\text {high }}=0.1289$, respectively. For the numerical simulation of the zero temperature $(T=0)$ system we took $12^{3} \cdot 24$ lattices, which turned out to be large enough for the present $L_{t}=2$ simulations. (Runs on $16^{3} \cdot 32$ are planned in near future [16].)

The numerical Monte Carlo simulations have been performed on the Quadrics Q16 machines at DESY. In order to decrease autocorrelations we applied a mixture of heatbath and overrelaxation algorithms with an optimized ratio, which depends on lattice sizes and bare parameters. An important improvement could be achieved by an overrelaxation algorithm in the Higgs field length $(\rho)$ variable which has been developed and tested by two of us 18. The heatbath algorithm for the gauge field can be taken over from pure gauge theory [19]. For the Higgs field one can start a heatbath algorithm at our small $\lambda$ values by generating an optimized Gaussian part of the single spin distribution and then correct by an accept-reject step for the remaining quartic piece [20]. Away from the critical $\kappa$ such combination of updating sweeps, which we call "complete sweep", almost entirely removes the autocorrelation. On our $12^{3} \cdot 24$ lattices the integrated autocorrelations for different characteristic quantities are between 2 and 20 complete sweeps. The smallest integrated autocorrelations are shown by Wilson loops, the largest ones by variables proportional to the length of the Higgs field. In the metastability region with tunneling between the two phases we still have the "supercritical slowing down", however, in the simulations presented here this does not play a rôle. In the two-coupling simulations of sections 3 and 4 the observed integrated autocorrelations were maximally of the order of 100 to 300 complete sweeps.

Performing 160000 complete sweeps on $12^{3} \cdot 24$ lattices and using an exponential + constant fit for the timeslice correlations of the quantities in (3) one obtains the following masses in lattice units:

$$
\begin{array}{rlrl}
a M_{H}^{l o w}=0.236(7), & & a M_{H}^{\text {high }}=0.262(9), \\
a M_{W}^{\text {low }}=1.059(24), & a M_{W}^{\text {high }}=0.427(8) .
\end{array}
$$

Here, as everywhere in this paper, statistical errors in last digits are given in parentheses. The estimates of the statistical errors always come from dividing our simulation data into 
independent subsets and performing the fits in these subsets in order to obtain the variance of the fit parameters. This procedure is particularly simple to implement on a Quadrics parallel computer, where in most cases several independent lattices are simulated. In our case we started Lüscher's random number generator [21] differently on each node, leading to statistically independent samples on the independent lattices.

In general we do not yet attempt to estimate our systematical errors, because they are presumably dominated by the present limitation to $L_{t}=2$ lattices for the high temperature simulations. In the continuum limit one has to consider $L_{t} \gg 1$, which is possible, but can be demanding [16].

For the mass ratio $R_{H W} \equiv M_{H} / M_{W} \equiv \sqrt{8 \lambda_{R}} / g_{R}(6)$ gives

$$
R_{H W}^{\text {low }}=0.222(12), \quad R_{H W}^{\text {high }}=0.614(32) .
$$

With $M_{W} \simeq 80 \mathrm{GeV}$ this means that the low point is at $M_{H} \simeq 18 \mathrm{GeV}$ and the high point at $M_{H} \simeq 49 \mathrm{GeV}$. On the $L_{t}=2$ lattices we have $a T_{c}=0.5$, therefore (6) also implies

$$
T_{c}^{l o w}=0.472(11) \cdot M_{W}, \quad T_{c}^{h i g h}=1.171(22) \cdot M_{W}
$$

The static potential has been extracted from $r \otimes t$ Wilson loops with $1 \leq r \leq 6$ and $1 \leq t \leq 12$ on $12^{3} \cdot 24$. We determined every such Wilson loop after transforming the gauge configuration to temporal gauge. The $t$-dependence has been fitted by three exponentials in order to determine the large- $t$ asymptotics. The potential can be well fitted by a Yukawapotential with lattice corrections, as discussed in [17]. Taking a form

$$
V(r)=-\frac{A}{r} e^{-M r}+C+D \cdot G(M, r)
$$

with $G(M, r)$ being the difference of lattice Yukawa-potential and continuum Yukawa potential, we obtained with $\chi^{2}$ of order one:

$$
\begin{gathered}
A^{\text {low }}=0.0336(3), \quad M^{\text {low }}=1.112(15), \quad C^{\text {low }}=0.066453(6), \quad D^{\text {low }}=0.0343(23), \\
A^{\text {high }}=0.03434(7), \quad M^{\text {high }}=0.4272(21), \quad C^{\text {high }}=0.090768(18), \quad D^{\text {high }}=0.0352(8) .
\end{gathered}
$$

The excellent agreement of the masses $M$ in (10) with $M_{W}$ in (6) and the nearly equality of the fit parameters $A$ and $D$ show that the static potential is well understood, and can be used for the definition of the renormalized gauge coupling. One could take for a definition $g_{R}^{2}=16 \pi A / 3$, but we prefer here not to use a global fit. Instead, we adopt for the definition of the renormalized gauge coupling a procedure similar to the one recently proposed in pure gauge theory [22]. Namely, starting from eq. (35) in ref. [17] we define an $r$-dependent renormalized gauge coupling, which we then interpolate to a distance $r=M_{W}^{-1}$. The result is, this time including also an estimate of the systematic errors as the last entry in parentheses:

$$
g_{R}^{2, l o w}=0.5476(5+75+10), \quad g_{R}^{2, h i g h}=0.5781(4+88+3) .
$$

The first two entries are the statistical errors from the Wilson loops and $M_{W}$, respectively. As one can see, the errors are dominated by the statistical errors of $M_{W}$ used as input in this analysis. This can be easily improved by better statistics, if later on required. Otherwise the errors are quite small, and the renormalization compared to the bare value $g^{2}=0.5$ is moderate. 


\section{Latent heat}

The pressure $(P)$ is continuous, therefore the latent heat (i. e. the discontinuity of the energy density $\Delta \epsilon)$ can be calculated from the discontinuity of the quantity $\delta \equiv \epsilon / 3-P$. Since $\delta$ is given by 23

$$
\delta=\frac{1}{3}\left(T L_{t}\right)^{4}\left\langle\frac{\partial \kappa}{\partial \tau} \cdot 8 L_{\varphi}-\frac{\partial \lambda}{\partial \tau} \cdot Q_{x}-\frac{\partial \beta}{\partial \tau} \cdot 6 P_{U}\right\rangle,
$$

where $\tau \equiv \log \left(a M_{W}\right)^{-1}$, and besides $L_{\varphi}$ in (1) we used

$$
Q_{x} \equiv\left(\rho_{x}^{2}-1\right)^{2}, \quad P_{U} \equiv 1-\frac{1}{2} \operatorname{Tr} U_{p l} .
$$

The vacuum contribution is not subtracted in (12), but in the latent heat it cancels and we obtain

$$
\frac{\Delta \epsilon}{T_{c}^{4}}=L_{t}^{4}\left\langle\frac{\partial \kappa}{\partial \tau} \cdot 8 \Delta L_{\varphi}-\frac{\partial \lambda}{\partial \tau} \cdot \Delta Q_{x}-\frac{\partial \beta}{\partial \tau} \cdot 6 \Delta P_{U}\right\rangle .
$$

The partial derivatives are taken here along the lines of constant physics (LCP's), which can be defined by keeping constant the value of the mass ratio $R_{H W}$ and the renormalized gauge coupling $g_{R}$. For weak bare couplings one can estimate the change of $g^{2}=4 / \beta$ and $\lambda_{0} \equiv \lambda /\left(4 \kappa^{2}\right)$ by integrating the one-loop renormalization group equations

$$
\begin{gathered}
\frac{d g^{2}(\tau)}{d \tau}=\frac{1}{16 \pi^{2}}\left[-\frac{43}{3} g^{4}+O\left(\lambda_{0}^{3}, \lambda_{0}^{2} g^{2}, \lambda_{0} g^{4}, g^{6}\right)\right] \\
\frac{d \lambda_{0}(\tau)}{d \tau}=\frac{1}{16 \pi^{2}}\left[96 \lambda_{0}^{2}+\frac{9}{32} g^{4}-9 \lambda_{0} g^{2}+O\left(\lambda_{0}^{3}, \lambda_{0}^{2} g^{2}, \lambda_{0} g^{4}, g^{6}\right)\right] .
\end{gathered}
$$

Starting from our low or high critical point of the $L_{t}=2$ lattice one can obtain the corresponding $L_{t}=3,4,5, \ldots$ points by integrating (15) from $\tau=0$ to $\tau=\log (3 / 2), \log (4 / 2), \log (5 / 2), \ldots$. For instance, the $L_{t}=3$ critical point corresponding to our low point $(\beta=8, \lambda=0.0001, \kappa=$ $0.12830)$ comes out to be at $(\beta=8.147, \lambda=0.000111)$ and the one corresponding to the high point $(\beta=8, \lambda=0.0005, \kappa=0.12887)$ at $(\beta=8.147, \lambda=0.000507)$.

Of course, we still need another equation for the change of the critical $\kappa=\kappa_{c}$. The critical hopping parameter can be determined by different methods. We found it convenient to apply a two-coupling method: on a long lattice in one spatial direction (say, z-direction) the lattice is subdivided into two equal halves with two different hopping parameters in such a way that the first half with $\kappa_{1}$ is in the symmetric phase, the second half with $\kappa_{2}$ in the Higgs phase. This means $\kappa_{1}<\kappa_{c}<\kappa_{2}$. To be sure that this situation is stable we require that both halves stay in their phases at least for 20 autocorrelation times. Going to larger lattices $\kappa_{1,2}$ can be chosen closer to $\kappa_{c}$ at the order of inverse lattice volume. From the $2 \cdot 16^{2} \cdot 128$ lattice at the low point and $2 \cdot 32^{2} \cdot 256$ lattice at the high point the best estimates are, respectively,

$$
\kappa_{c, L_{t}=2}^{\text {low }}=0.12830(5), \quad \kappa_{c, L_{t}=2}^{\text {high }}=0.12887(1) .
$$

These are rather well reproduced, together with the volume dependence on smaller lattices, by the critical $\kappa$ obtained from the one loop gauge invariant effective potential of the length square of the scalar field $V_{\text {eff }}\left(\rho^{2}\right)$ [24, 25, 16]. Therefore one can use $V_{\text {eff }}\left(\rho^{2}\right)$ for an estimate of $\kappa_{c, L_{t}=3,4,5, \ldots}$. By a quadratic interpolation of the $L_{t}=2,3,4$ critical points one obtains the following estimates for the derivatives of $\kappa$ along the LCP's:

$$
\frac{\partial \kappa^{\text {low }}}{\partial \tau}=-0.00064(6), \quad \frac{\partial \kappa^{h i g h}}{\partial \tau}=-0.00111(13)
$$


The discontinuities in (14) can be determined at the low point on large enough lattices directly at $\kappa=\kappa_{c}$, because the metastability is very strong and the configurations stay for a very long time in the phase they started from. At the high point a linear extrapolation can be used from the intervals $\kappa \leq \kappa_{1}$ and $\kappa \geq \kappa_{2}$, where no tunnelings to the other phase occur. On our $2 \cdot 16^{2} \cdot 128$ and $2 \cdot 32^{2} \cdot 256$ lattices at the low and high points, respectively, the finite volume corrections of order $\left(L_{x} L_{y} L_{z}\right)^{-1}$ are already smaller than the indicated statistical errors:

$$
\begin{gathered}
\Delta L_{\varphi}^{\text {low }}=-21.84(4), \quad \Delta Q_{x}^{\text {low }}=-643(2), \quad \Delta P_{U}^{\text {low }}=0.010557(15), \\
\Delta L_{\varphi}^{\text {high }}=-1.026(12), \quad \Delta Q_{x}^{\text {high }}=-8.02(13), \quad \Delta P_{U}^{\text {high }}=0.000629(8) .
\end{gathered}
$$

Combining this with (14)-(15) we arrive at

$$
\left(\frac{\Delta \epsilon}{T_{c}^{4}}\right)^{\text {low }}=1.68(17), \quad\left(\frac{\Delta \epsilon}{T_{c}^{4}}\right)^{\text {high }}=0.125(19) .
$$

These results have been obtained on $L_{t}=2$ lattices alone. In the continuum limit $L_{t} \rightarrow \infty$, therefore later on one has to improve them by simulations on finer lattices with $L_{t}>2$ [16]. For a first qualitative comparison with two-loop resummed perturbation theory [5] see fig. 1 .

\section{Interface tension}

Besides the latent heat another important physical quantity characterizing the strength of the phase transition is the interface tension $\sigma$ between the two phases, which can be determined on the lattice in several different ways. In the present letter we concentrate on the two-coupling method proposed by Potvin and Rebbi [26], where an interface pair is enforced between the two halves of the periodic lattice. Since we have three bare parameters, in principle one can choose any of them (or some combination of them) to be different in the two halves. As in the latent heat the contribution of the $\varphi$-link $L_{\varphi}$ dominates, we decided to split the value of $\kappa$ multiplying $L_{\varphi}$ in the action. On an $L_{t} \cdot L_{x} \cdot L_{y} \cdot L_{z}$ lattice with $L_{z} \gg L_{x, y, t}$, and in our case $L_{t}=2, L_{x}=L_{y}$, the two halves in the $z$-direction have hopping parameters $\kappa_{1,2}$ which are slightly below and above the critical one: $\kappa_{1}<\kappa_{c}<\kappa_{2}$. The interface tension between the states with $\kappa_{1}$ and $\kappa_{2}$ is given in lattice units by

$$
a^{3} \sigma\left(\kappa_{1}, \kappa_{2}\right)=L_{z}\left\{\int_{\kappa_{1}}^{\kappa_{2}} d \kappa L_{\varphi}^{(1)}\left(\kappa, \kappa_{2}\right)-\int_{\kappa_{1}}^{\kappa_{2}} d \kappa L_{\varphi}^{(2)}\left(\kappa_{1}, \kappa\right)\right\},
$$

where $L_{\varphi}^{(1,2)}\left(\kappa, \kappa^{\prime}\right)$ denote the expectation values of the $\varphi$-link in the two halves if the hopping parameters are $\kappa$ and $\kappa^{\prime}$, respectively.

In order to obtain the physically interesting interface tension one has, of course, to perform the limits $L_{x, y, z} \rightarrow \infty$ and $\Delta \kappa \equiv \kappa_{2}-\kappa_{c}=\kappa_{c}-\kappa_{1} \rightarrow 0$. For a given lattice extension, however, $\Delta \kappa$ cannot be arbitrarily small, because if the difference in free energy density becomes small tunneling into the other phase can occur and the interfaces disappear. The presence of the interfaces can, however, be monitored to ensure the applicability of (20). For small $\Delta \kappa$ the integrals in (20) can be well approximated by the average of the values of the integrand at the two end points. Besides, for equal arguments we obviously have $L_{\varphi}^{(1)}(\kappa, \kappa)=L_{\varphi}^{(2)}(\kappa, \kappa)$. This gives

$$
a^{3} \sigma \simeq L_{z} \Delta \kappa\left(L_{\varphi}^{(1)}\left(\kappa_{1}, \kappa_{2}\right)-L_{\varphi}^{(1)}\left(\kappa_{1}, \kappa_{1}\right)+L_{\varphi}^{(2)}\left(\kappa_{2}, \kappa_{2}\right)-L_{\varphi}^{(2)}\left(\kappa_{1}, \kappa_{2}\right)\right) .
$$


In the limit $\Delta \kappa \rightarrow 0$ the contributions with equal hopping parameters in the two halves do not contribute. The non-zero contributions can be determined by the expansion

$$
\begin{aligned}
& L_{\varphi}^{(1)}\left(\kappa_{1}, \kappa_{2}\right)=\frac{-c_{1}}{\kappa_{1}-\kappa_{c}}+b_{1}+a_{1}\left(\kappa_{1}-\kappa_{c}\right)+O\left(\kappa_{1}-\kappa_{c}\right)^{2}, \\
& L_{\varphi}^{(2)}\left(\kappa_{1}, \kappa_{2}\right)=\frac{-c_{2}}{\kappa_{2}-\kappa_{c}}+b_{2}+a_{2}\left(\kappa_{2}-\kappa_{c}\right)+O\left(\kappa_{2}-\kappa_{c}\right)^{2} .
\end{aligned}
$$

This formula replaces (20) in the region where $\sigma\left(\kappa_{1}, \kappa_{2}\right)$ depends linearly on $\kappa_{1,2}$. For $\Delta \kappa \rightarrow 0$ a finite volume estimator of the interface tension is

$$
a^{3} \hat{\sigma}=L_{z}\left(c_{1}+c_{2}\right)
$$

The advantage compared to (20) is that no numerical evaluation of integrals is necessary.

In our numerical simulations several lattice sizes up to $2 \cdot 16^{2} \cdot 128$ at the low point and up to $2 \cdot 32^{2} \cdot 256$ at the high point were exploited. In fact, the very long extensions in the $z$-direction have been chosen just for the purpose of also determining the interface tension. The measured $L_{\varphi}^{(1,2)}$ values were fitted in a carefully chosen $\kappa$-interval around the critical points in (16) by the three-parameter forms in (22). At the low point we used the intervals $0.1279 \leq \kappa_{1} \leq 0.1282$ and $0.1284 \leq \kappa_{2} \leq 0.1287$ with seven points each. At the high point we used $0.12880 \leq \kappa_{1} \leq 0.12886$ and $0.12888 \leq \kappa_{2} \leq 0.12894$ with seven points each. The number of complete sweeps for measurements with an optimized ratio of heatbath to overrelaxation was between 20000 and 40000 per point. The obtained result from the fits in different subintervals is:

$$
\left(\frac{\hat{\sigma}}{T_{c}^{3}}\right)^{\text {low }}=0.84(16), \quad\left(\frac{\hat{\sigma}}{T_{c}^{3}}\right)^{\text {high }}=0.008(2) .
$$

Here $a T_{c}=\frac{1}{2}$ has been used in order to convert from lattice to physical units. About half of the indicated errors come from statistics. The other half is a crude estimate of systematic errors, which is due to the choice of subintervals for $\kappa$. In general, choosing a subinterval closer to $\kappa_{c}$ gives smaller statistical errors and also somewhat smaller $c_{1,2}$. The quoted results are obtained from the more distant subintervals.

This dependence on the chosen fit interval is a kind of rounding-off effect which is partly due to the interaction of the two interfaces. A similar dependence of $\sigma\left(\kappa_{1}, \kappa_{2}\right)$ on $\kappa_{1,2}$ appears in (20) if $\kappa_{1,2}$ are too close to $\kappa_{c}$. This $\kappa$-dependence is expected to be substantially reduced for $L_{z} \rightarrow \infty$ when the two interfaces do not interact. Of course, the transversal extensions $L_{x, y}$ also have to be taken to infinity. In our case comparisons to smaller lattices show in both points that within the given statistical errors $L_{x}=L_{y}$ are large enough. In principle, if $L_{x, y, z}$ are larger one can consider smaller $\Delta \kappa$ values, but this is beyond the scope of the present analysis.

We also tried several other methods for the determination of $\sigma$. The results are in general qualitatively similar. A more detailed evaluation of the different methods will be included in a subsequent publication [16]. A comparison of (24) with two-loop resummed perturbation theory [5] is shown in fig. 2. 


\section{Discussion}

The outcome of our numerical studies on $L_{t}=2$ lattices is a qualitative agreement between twoloop resummed perturbation theory and four dimensional lattice simulations in the investigated range of Higgs boson masses, below $50 \mathrm{GeV}$. Note that at $M_{H} \simeq 18 \mathrm{GeV}$ the agreement between our data and perturbation theory becomes even better if, instead of $T_{c}, M_{H}$ is used to set the scale. Both latent heat and interface tension show that the first order symmetry restoring phase transition becomes substantially weaker for increasing Higgs boson mass (see eqs. (19) and (24)). As it has been emphasized throughout this paper, although the $L_{t}=2$ results may very well be qualitatively right, for a quantitative determination of the properties of the phase transition further numerical simulations on $L_{t}>2$ lattices are necessary.

\section{Acknowledgements}

We thank our colleagues W. Buchmüller, A. Hebecker, M. Lüscher and R. Sommer for essential comments and proposals during the course of this work. F. Cs. and Z. F. were partially supported by Hung. Sci. Grant under Contract No. OTKA-F1041/3-2190.

\section{References}

[1] D.A. Kirzhnitz, JETP Lett. $\underline{15}$ (1972) 529;

D.A. Kirzhnitz, A.D. Linde, Phys. Lett. B72 (1972) 471; Ann. Phys. 101 (1976) 195.

[2] M.E. Carrington, Phys. Rev. D45 (1992) 2933.

[3] W. Buchmüller, Z. Fodor, T. Helbig, D. Walliser, preprint DESY-93-021, Ann. Phys. to appear.

[4] P. Arnold, O. Espinosa, Phys. Rev. D47 (1993) 3546.

[5] Z. Fodor, A. Hebecker, preprint DESY-94-025.

[6] A. D. Linde, Phys. Lett. $\underline{96 B}$ (1980) 289.

[7] P. H. Damgaard, U. M. Heller, Nucl. Phys. B294 (1987) 253.

[8] H. G. Evertz, J. Jersák, K. Kanaya, Nucl. Phys. B285 [FS19] (1987) 229.

[9] B. Bunk, E.-M. Ilgenfritz, J. Kripfganz, A. Schiller, Phys. Lett. B284 (1992) 371; Nucl. Phys. B403 (1993) 453.

[10] K. Kajantie, K. Rummukainen, M.E. Shaposhnikov, Nucl. Phys. B407 (1993) 356.

[11] A. Jakovác, K. Kajantie, A. Patkós, preprint HU-TFT-94-01. 
[12] V.A. Kuzmin, V.A. Rubakov, M.E. Shaposhnikov, Phys. Lett. B155 (1985) 36.

[13] M.E. Shaposhnikov, Nucl. Phys. B287 (1987) 757.

[14] A.G. Cohen, D.B. Kaplan, A.E. Nelson, Annu. Rev. Nucl. Part. Sci. $\underline{43}$ (1993) 27.

[15] K. Farakos, K. Kajantie, K. Rummukainen, M.E. Shaposhnikov, preprints CERNTH.6973/94, CERN-TH.7244/94.

[16] F. Csikor, Z. Fodor, J. Hein, K. Jansen, A. Jaster, I. Montvay, in preparation.

[17] W. Langguth, I. Montvay, P. Weisz, Nucl. Phys. B277 (1986) 11.

[18] Z. Fodor, K. Jansen, preprint DESY-94-046, Phys. Lett. to appear.

[19] A.D. Kennedy, B.J. Pendleton, Phys. Lett. B156 (1985) 393.

[20] B. Bunk, private communication.

[21] M. Lüscher, Comp. Phys. Comm. $\underline{79}$ (1994) 100.

[22] R. Sommer, Nucl. Phys. B411 (1994) 839.

[23] I. Montvay, G. Münster, Quantum Fields on a Lattice, Cambridge University Press, 1994.

[24] M. Lüscher, unpublished notes (1988).

[25] W. Buchmüller, Z. Fodor, A. Hebecker, preprint DESY-94-043, Phys. Lett. to appear.

[26] J. Potvin, C. Rebbi, Phys. Rev. Lett. $\underline{62}$ (1989) 3062;

S. Huang, J. Potvin, C. Rebbi, S. Sanielevici, Phys. Rev. D42 (1990) 2864, errata: D43 (1991) 2056.

\section{Figure captions}

Fig. 1. Comparison of the results of Monte Carlo simulations with two-loop resummed perturbation theory [5]. The latent heat $\Delta \epsilon$ is shown as a function of the Higgs boson mass. The two curves correspond to the two slightly different gauge couplings in (11): the full line to the low value, the dashed line to the high one.

Fig. 2. The same as fig. 1 for the interface tension $\sigma$. 
This figure "fig1-1.png" is available in "png" format from: http://arxiv.org/ps/hep-lat/9405021v2 
Figure 1.

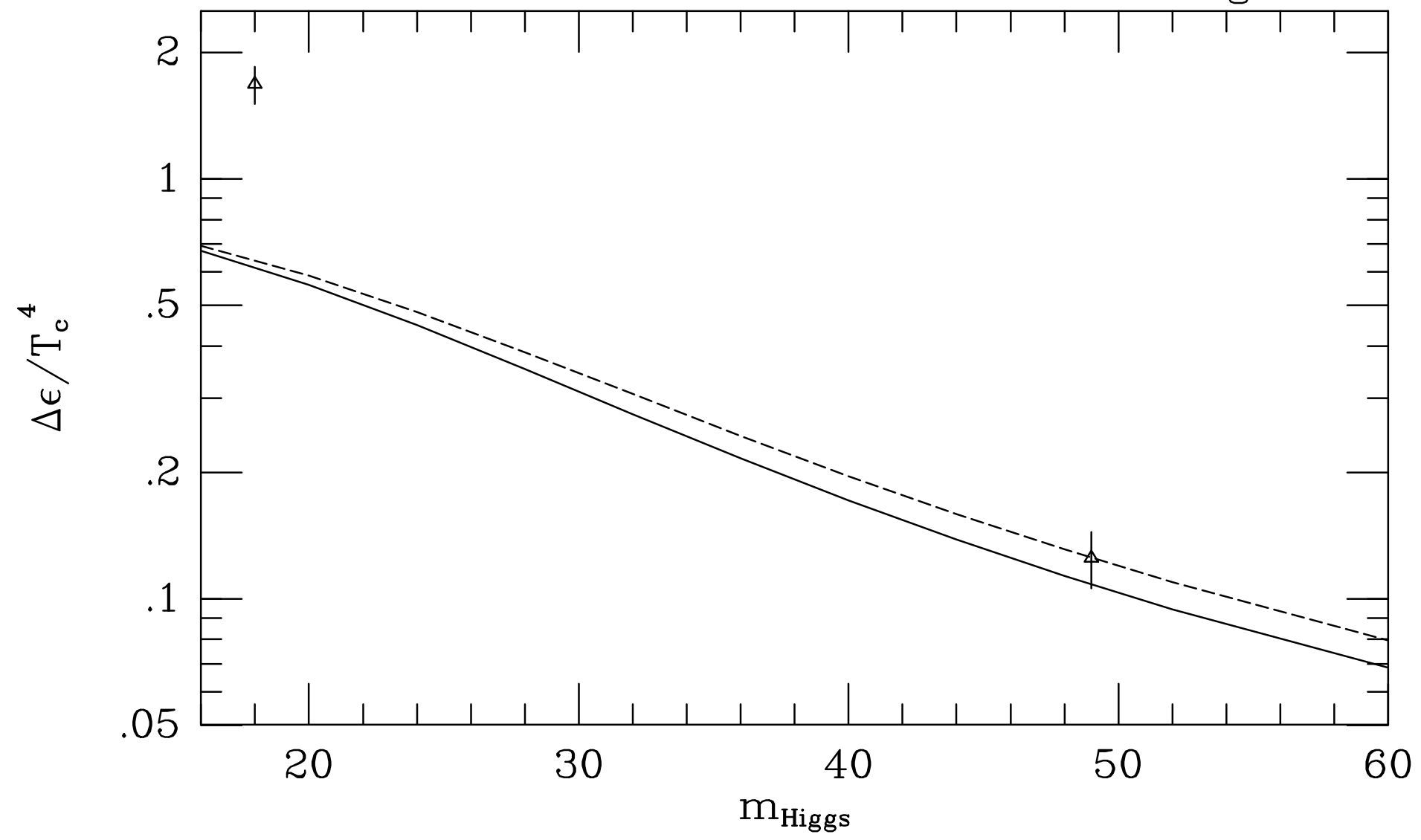

Figure 2.

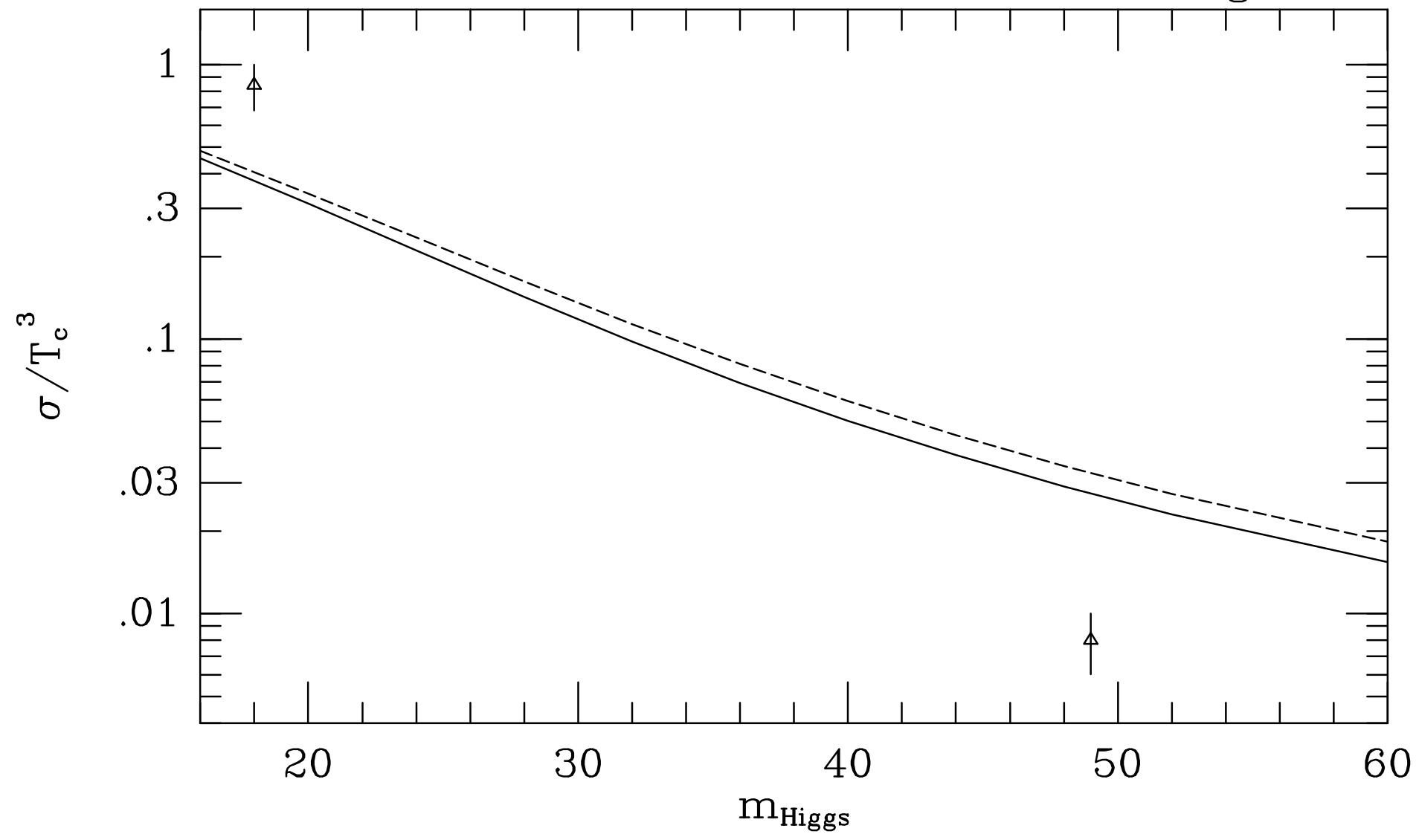

